\title{
Pensamiento aleatorio para tercero primaria rural durante la pandemia por covid-19
}

\section{Random thinking for third-grade of rural primary school during covid-19 pandemic}

\author{
Elizabeth Cuevas Solano ${ }^{1}$ \\ Ivonne Zoraya Muñoz Prieto² \\ Robinson Julian Serna ${ }^{3}$ \\ UPTC
}

\section{RESUMEN}

En respuesta al reto educativo emergente debido a la pandemia por la Covid-19 y para mitigar algunas debilidades evidenciadas en las pruebas Saber (componente aleatorio) para grado tercero primaria (ICFES, 2018) y en el estudio SERCE (Ruiz López, 2015) realizado por la UNESCO, la presente investigación

\footnotetext{
Institución Educativa Andrés Bello, Arauquita,

Colombia. elizabeth.cuevas@uptc.edu.co

+573209496488

2 Instituto Arturo Ramírez Montufar (IPARM), Bogotá,

Colombia.ivmunozp@unal.edu.co+573195284738

3 Escuela de Matemáticas y Estadística,

Universidad Pedagógica y Tecnológica de Colombia, Tunja,

Colombia. robinson.serna@uptc.edu.co +573143339488
}

describe y analiza la experiencia de implementar el uso de recursos virtuales en el proceso de enseñanza-aprendizaje de la probabilidad intuitiva en estudiantes de grado tercero de primaria de una escuela rural durante el periodo de confinamiento debido a la pandemia por la Covid-19 en Colombia (Wikipedia, 2021) El estudio se enmarca dentro del paradigma cualitativo de tipo descriptivo e implementa la Investigación Acción-Participativa (IAP). Respecto a la metodología investigativa, una entrevista abierta de entrada permitió describir los pre-saberes de los estudiantes en relación con la probabilidad intuitiva, enseguida, se diseñó e implementó una secuencia didáctica (Cuevas, 2020) y, finalmente, una entrevista 
abierta de salida permitió evaluar el impacto y la experiencia vivida en la etapa anterior.

En el análisis de resultados y apelando a la experiencia vivida en la emergencia sanitaria por la Covid-19, se establecen sugerencias acerca del uso de estrategias virtuales para la enseñanza de la probabilidad intuitiva en edades tempranas y durante situaciones de emergencia en zonas rurales. En este contexto, los resultados investigativos evidencian una incidencia positiva en la formación de capacidades para la toma de decisiones en situaciones cotidianas que involucran cierto grado de incertidumbre.

PALABRAS CLAVE: probabilidad intuitiva, pandemia por la Covid-19, educación rural, recursos virtuales, material de apoyo didáctico.

\section{ABSTRACT}

According to the emerging educational challenge due to the Covid-19 pandemic and to mitigate some weaknesses evidenced in the Saber tests (random component) for third-grade primary (ICFES, 2018) and the SERCE study (Ruiz López, 2015 ) by UNESCO, this research describes and analyzes the experience of implementing the use of virtual resources in the teaching-learning process of statistical probability in third-grade students of a rural school during the period of confinement due to the Covid-19 pandemic in Colombia (Wikipedia, 2021). The study is framed within the qualitative descriptive paradigm and implements Participatory Action Research (PAR). In the research phases, an open entry interview allowed describing the students' pre-knowledge about intuitive probability. Then, a didactic sequence was designed and implemented (Cuevas, 2020). Finally, an open exit interview allowed evaluating the impact of the experience lived in the previous stage.

In the analysis of the results and according to the experience lived in the health emergency caused by Covid 19, we establish some suggestions about virtual strategies for teaching intuitive probability at an early age and during emergencies in rural areas. In this context, the results evidence a positive impact on the training of abilities to make decisions in everyday situations that involve a certain degree of uncertainty.

KEYWORDS: intuitive probability, Covid-19 pandemic, rural education, virtual resources, didactic support material.

\section{INTRODUCCIÓN}

El estado de emergencia a causa de la pandemia por la Covid-19 ha demostrado que la enseñanza en este tipo de circunstancias no es fácil. Los profesores han hecho un mayor uso de la educación a distancia y los estudiantes han tenido que adaptarse. El clásico estudio presencial no ha sido posible; sin embargo, en la mayoría de los casos, es reemplazado por el uso de algunas tecnologías de la información y la comunicación (TIC) disponibles. En el caso rural colombiano, las dificultades se incrementan debido al bajo índice de conectividad en estas zonas y la poca disponibilidad de aparatos tecnológicos.

Por otra parte, uno de los retos educativos es la enseñanza de la estadística en edades tempranas. A nivel colombiano, las pruebas Saber, implementadas por el Instituto Colombiano para la Evaluación de la Educación (ICFES, 2018), indican algunas dificultades de aprendizaje de las nociones intuitivas de azar y probabilidad que requieren ser superadas; problemática que se relaciona directamente con los resultados del estudio SERCE, de la Organización de las Naciones Unidas para la Educación, la Ciencia y la Cultura (UNESCO), el cual afirma que aproximadamente el $16 \%$ del tiempo de enseñanza de las matemáticas se dedica a la estadística, tanto en $3^{\circ}$ como en $6^{\circ} \mathrm{y}$, que los contenidos que más se 
estudian en estos cursos son los referidos a la elaboración e interpretación de tablas y gráficos, dejando para cursos posteriores nociones de probabilidad y la resolución de problemas usando estadística (Ruiz López, 2015, pág. p.103). En consecuencia, el interrogante que orienta la investigación es ¿Cómo fortalecer el pensamiento aleatorio mediante una estrategia didáctica basada en recursos virtuales que sea propicia para el tiempo de confinamiento por la pandemia debido a la Covid-19?

El estudio tiene como base algunos antecedentes investigativos que dan solidez a la investigación, relacionando temáticas de azar, percepción de aleatoriedad y probabilidad, sus significados en estudiantes de educación básica, la estadística por proyectos, la didáctica de la matemática y el uso de los recursos TIC en su enseñanza. Así mismo, se tienen en cuenta los aportes realizados por autores como (Gómez Torres, Contreras, \& Batanero, 2015), quienes explican los significados de la probabilidad en la educación primaria, exponiendo cada uno de los pasos a seguir para enseñar a diferenciar los fenómenos aleatorios y deterministas. En referencia a la didáctica de la probabilidad (Burbano Pantoja, Valdivieso Miranda, \& Aldana Bermúdez, 2017) expresan que "Es conveniente que el profesor inicie a sus estudiantes con las ideas intuitivas durante los primeros años de escolaridad y prosiga de forma gradual con las concepciones clásica y frecuencial con el fin de hacer una aproximación hacia la axiomática en los últimos grados de la educación media". (p.272).

La presente investigación está enfocada en el desarrollo del pensamiento aleatorio, particularmente en la enseñanza de nociones de probabilidad intuitiva. Por lo general, se tiende a pensar que probabilidad es lo mismo que estadística; sin embargo, (Jiménez Vargas, 2014) expone esta aclaración con base a autores como J. Díaz Godino (1996), quien afirma que "la probabilidad aporta una forma de medir la incertidumbre y que es la base de la estadística, lo que implica que, para tener una adecuada comprensión de los modelos estadísticos, se tenga que tener un amplio conocimiento sobre la probabilidad" (p.7). Para tal fin, se resaltan aspectos esenciales de la pedagogía activa en la práctica educativa, con el propósito de enriquecer y estructurar de manera significativa las nociones de probabilidad en estudiantes de básica primaria del grado tercero. Es de reconocer que en el presente siglo ya se tiene en cuenta la importancia de la inclusión de la probabilidad y la estadística en el currículo escolar desde la básica primaria. Algunos estudios comprueban sus implicaciones en distintas disciplinas, ya que por medio de esta se fortalece la comprensión y el razonamiento crítico. En el congreso "las nuevas metodologías en la enseñanza y el aprendizaje de las matemáticas" (Consejería de Educación de la Junta de Castilla y León, 2015) se referencia la teoría del pensamiento estadístico planteada por (Gaise, Franklin y cols. 2007) quien denota la omnipresencia de la variabilidad en la matemática. “... reconocer que la variabilidad es ubicua en muchos fenómenos cotidianos. La variabilidad es la esencia de la Estadística como disciplina y no puede ser entendida solo mediante estudio y lectura, sino que debe ser experimentada" (p.72). Aspecto pertinente en el planteamiento de la investigación debido a la importancia de promover metodologías dinámicas y participativas que le permitan al estudiante ser un sujeto activo en su proceso de aprendizaje.

En consecuencia, en la fase de trabajo de campo se diseña e implementa una secuencia didáctica (Cuevas, 2020) que cuenta con un enfoque socio-formativo pasando de una perspectiva de contenidos a procesos dinamizadores de formación en los que se establecen unos objetivos de aprendizaje para el desarrollo del pensamiento aleatorio. Para su diseño se sigue 
el formato sugerido por (Tobón Tobón, Pimienta Prieto, \& García Fraile, 2010) quienes presentan una metodología estándar que permite realizar la adaptación respectiva acorde a los problemas del contexto y el proceso meta cognitivo de los estudiantes del grado tercero. Esta secuencia didáctica está articulada al modelo TPACK (Technological Pedagogical Content Knowledge) Molina et al. (2019) a través de Objetos Virtuales de Aprendizaje (OVA), advirtiéndose que, en el confinamiento por la pandemia actual, las TIC brindan herramientas con diversas aplicaciones que facilitan la orientación académica en casa. Acerca del modelo TPACK y de la incorporación de las TIC en educación, Molina et al. (2019) resaltan la integración de las TIC en el campo investigativo como un recurso que brinda diversas herramientas para innovar en los procesos de enseñanza y aprendizaje, siendo necesario como docentes apropiarnos de su uso. Los referidos autores exponen que "...en los últimos cinco años, la preocupación por mejorar la educación a partir de las nuevas tecnologías se incrementa debido a las dinámicas propias de lo que ahora se denomina la era digital" (p.11).

\section{METODOLOGÍA}

\subsection{Enfoque y tipo de Investigación.}

La investigación pertenece al enfoque cualitativo, de tipo descriptivo enmarcado en el diseño de campo (Hernández Sampieri, Fernández Collado, \& Baptista Lucio, 2004). En concordancia, el tipo de investigación es la Investigación Acción-Participativa, ya que sus características metodológicas permiten llevar a cabo cada fase investigativa en afinidad con los objetivos propuestos. La IAP "supone la simultaneidad del proceso de conocer y de intervenir, e implica la participación de la misma gente involucrada en el programa de estudio y de acción" (Ander-Egg, 2003, pág. 33). De manera que, la muestra de estudiantes tomada como objeto de estudio son agentes activos que contribuyen a conocer y transformar su realidad, recalcando aún más ese rol de sujetos activos y autónomos en su proceso de formación dadas las circunstancias de aprendizaje en casa debido a la pandemia por la Covid-19. (Ander-Egg, 2003) reafirma lo expuesto anteriormente, al expresar que "El proceso del método y el procedimiento propio de la IAP suscita en quienes participan una toma de conciencia de sus propias capacidades de actuar y movilizarse para lograr una transformación y un mejoramiento de su propia situación" (p. 34).

\subsection{Muestra y Técnicas de recolección de la información.}

La población seleccionada para la realización del estudio corresponde a una muestra de 19 estudiantes que cursan el grado tercero de educación básica primaria, en la institución educativaAndrésBello delmunicipio deArauquita. Las técnicas de recolección de información a implementar son la entrevista semi-estructurada y diario de campo, instrumentos que abordan aspectos esenciales del trabajo y permiten dar respuesta a la pregunta orientadora.

\subsection{Fases de Investigación.}

Este estudio sigue una secuencia metodológica distribuida de manera secuencial, iniciando con una entrevista semi-estructurada que permitió diagnosticar las dificultades que presentan los estudiantes en el desarrollo del pensamiento aleatorio. Para realizar dicha entrevista se utilizó la aplicación digital Whatsapp como herramienta virtual, siendo esta, una estrategia facilitadora para llevar a cabo la referida etapa. Esta fase diagnóstica fue el derrotero para diseñar y aplicar una secuencia didáctica titulada "Una puerta abierta a la aventura de la probabilidad" (Cuevas, 2020) estando articulada al modelo TPACK con el objetivo de potenciar temáticas de azar y probabilidad. Mishra y Koehler (2006) como se citó por Molina et al. (2019) explican el referido modelo analizando los procesos de integración de las TIC en la práctica pedagógica. 
"El modelo TPACK no corresponde a un modelo creado, sino al estudio de los factores siempre presentes en los procesos de integración de las TIC en la educación" (p.13). Es este orden de ideas, es preciso aclarar que el trabajo de campo llevado a cabo mediante la secuencia didáctica, dada la situación de contingencia mencionada anteriormente, se realizó usando recursos digitales mediante una página Web, Cuevas Solano, E. (2020) para su exploración y desarrollo, además de diseñarse su presentación en medio físico, elaborando una cartilla didáctica entregada a cada estudiante como apoyo para su desarrollo en casa, siendo esta una estrategia planteada como apoyo al recurso virtual, en vista de la limitada conectividad que tienen los estudiantes en la ruralidad.

La fase final corresponde a la correlación descriptiva de los resultados obtenidos a partir de la incidencia de los recursos implementados para potenciar el desarrollo del pensamiento aleatorio, estando, los estudiantes de tercero primaria, en la capacidad de brindar soluciones razonables a problemas o situaciones de su contexto en los que no hay una solución clara o segura, comprendiendo las nociones de probabilidad intuitiva.

\section{EXPERIENCIA VIVIDA EN TIEMPO DE PANDEMIA POR COVID-19}

Es de resaltar que el proceso de articulación de las TIC en la educación es un tema fuertemente estudiado; sin embargo, en el contexto rural colombiano su uso ha sobresalido en tiempo del confinamiento por pandemia debido a la Covid-19, situación que ha permitido apreciar tanto a estudiantes como a docentes experiencias enriquecedoras que los ha llevado a la auto capacitación en la utilización de recursos virtuales, aplicaciones y plataformas digitales. En la experiencia vivida con los estudiantes objeto de estudio, dada la limitada conectividad a internet y la débil señal del teléfono móvil, surgió el interrogante ¿Cómo generar el acompañamiento a los estudiantes que no pueden conectarse de manera remota? se optó como mejor estrategia de comunicación, el uso de la aplicación Whatsapp debido a que los padres de familia o acudientes no contaban con muchos dispositivos electrónicos para acceder a internet, siendo el teléfono inteligente la mejor opción para muchas familias rurales en las que con un teléfono móvil y la aplicación Whatsapp entre uno y tres estudiantes accedían a sus aulas virtuales, bien conocidas como "Grupos de Whatsapp". Sumado a ello, se diseñó una página web en la que se encontraban vídeos, audios, presentaciones interactivas y actividades de gamificación a las que podían acceder en el momento que dispusieran de conectividad; por otra parte, para contrarrestar la problemática del limitado acceso a internet, se implementó el uso de material impreso con el contenido digital desarrollado, la cartilla "una puerta abierta a la aventura de la probabilidad", que sirvió de apoyo a los estudiantes y a los padres de familia, que de diferentes maneras asumieron un rol activo en los procesos de aprendizaje. La implementación de este material impreso fue fundamental en la ruralidad, siendo los recursos virtuales material de apoyo para afianzar y motivar la participación activa en el proceso de aprendizaje.

En este escenario, se observó en la fase diagnóstica de la investigación la poca relación que tenían los estudiantes con la virtualidad, situación que influyó en que presentaran cierto nerviosismo y timidez en el desarrollo de la entrevista semi-estructurada de entrada que se realizó por video llamada a través de Whatsapp. Sin embargo, con la implementación de la secuencia didáctica, los estudiantes se familiarizaron con términos que encontraban desconocidos como link, página web y juegos en línea a través de actividades de gamificación, estrategias que influyeron positivamente para potenciar la motivación y facilitar la comprensión de situaciones en las que estaba presente el azar y la probabilidad. Para los estudiantes 
objeto de estudio, el aprendizaje desde la virtualidad pasó de ser algo desconocido a ser una estrategia atractiva y dinámica. Este nuevo panorama incidió en que en el desarrollo de la entrevista semiestructurada de salida se contará con mayor participación, observándose la superación del nerviosismo inicial, así mismo se dio una mejor comprensión de las situaciones problema planteadas.

\section{DISCUSIÓN DE RESULTADOS}

La tabla 1 resume el nivel de desempeño de los estudiantes de la muestra, denotados con los ítems $1,2, \ldots, 5$. Se realizaron dos entrevistas, una de entrada (prueba diagnóstica) y una de salida que evalúa el impacto de utilizar la secuencia didáctica diseñada. Los ítems evaluados corresponden a las evidencias de aprendizaje relacionados con el DBA (Derecho Básico de Aprendizaje) número 11 del grado tercero establecido por el Ministerio de Educación Nacional (Colombia, 2016).

Tabla 1. Items establecidos para definir el nivel de desempeño.

\begin{tabular}{|l|l|}
\hline Ítem 1 & $\begin{array}{l}\text { Comprensión del concepto de } \\
\text { probabilidad a partir de la cotidianidad. }\end{array}$ \\
\hline Ítem 2 & $\begin{array}{l}\text { Establece la probabilidad de } \\
\text { ocurrencia de eventos simples en una } \\
\text { escala cualitativa (imposible, poco } \\
\text { probable, muy probable). }\end{array}$ \\
\hline Ítem 3 & $\begin{array}{l}\text { Identifica a qué hace referencia } \\
\text { el concepto del azar a partir de } \\
\text { situaciones del contexto. }\end{array}$ \\
\hline Ítem 4 & $\begin{array}{l}\text { Expone ejemplos que se atribuyen a } \\
\text { una situación de azar. }\end{array}$ \\
\hline Ítem 5 & $\begin{array}{l}\text { Identifica si una situación es aleatoria } \\
\text { o no. }\end{array}$ \\
\hline
\end{tabular}

Fuente (Elaboración propia)

El nivel de desempeño se sitúa en una escala valorativa de uno a cinco de acuerdo a la siguiente tabla:
Tabla 2. Escala valorativa definida para establecer el nivel de desempeño de los estudiantes.

\begin{tabular}{|l|l|}
\hline $\begin{array}{l}\text { Nivel de } \\
\text { desempeño }\end{array}$ & Descripción \\
\hline 1 & $\begin{array}{l}\text { Indica no tener una respuesta. } \\
\text { pero su respuesta no es } \\
\text { completamente acertada. }\end{array}$ \\
\hline 2 & $\begin{array}{l}\text { Responde acertadamente } \\
\text { perono explica correctamente } \\
\text { su respuesta } \\
\text { interpretativo). }\end{array}$ \\
\hline 4 & $\begin{array}{l}\text { Responde acertadamente } \\
\text { y explica correctamente } \\
\text { su respuesta (Nivel } \\
\text { argumentativo). }\end{array}$ \\
\hline 5 & $\begin{array}{l}\text { Responde acertadamente, } \\
\text { explica su respuesta y } \\
\text { plantea otros ejemplos (Nivel } \\
\text { propositivo). }\end{array}$ \\
\hline
\end{tabular}

Fuente (Elaboración propia)

En relación al ítem 1, respecto a la prueba diagnóstica, el $94.7 \%$ de los entrevistados se ubican en el nivel de desempeño uno y dos, y el $5.3 \%$ restante se ubica en el nivel tres. En contraste, la entrevista de salida indica que el $63.1 \%$ de los estudiantes se ubican en el nivel cinco y el porcentaje restante en los niveles tres y cuatro. Se evidencia que, aunque el concepto intuitivo de probabilidad no es parte de los pre-saberes de la mayoría de estudiantes, el uso de la secuencia didáctica diseñada influye notablemente en la comprensión de este concepto y que los estudiantes asimilan mejor el concepto cuando se explica mediante situaciones cotidianas, por ejemplo, algunos de los estudiantes aseguraron que en la vereda de la Paz cuando llueve fuertemente es probable que se queden sin luz, situación que se presenta regularmente en su contexto. Una actividad incluida en la secuencia didáctica que se puede considerar "exitosa" en el sentido de promover la 
comprensión de la noción de probabilidad para estudiantes de tercero primaria se relaciona con la construcción y uso de material concreto, donde los estudiantes trabajaron con un cubo de colores (denominado dispositivo aleatorio) que ellos construyeron previamente. En la actividad, se propusieron situaciones en las que los niños y niñas podían determinar la probabilidad de un evento; por ejemplo, si el cubo tiene tres caras rojas, dos azules y una cara amarilla, al lanzar el cubo se pueden hacer preguntas como:

(a) ¿Qué color tiene más probabilidad de salir?

(b) ¿Qué color tiene menos probabilidad de salir?

\section{(c) ¿Es probable obtener el color verde?}

En cuanto al concepto de azar, inicialmente el $68,42 \%$ de los entrevistados expresan desconocer el término "azar", el 21,05\% respondió a la pregunta ¿Qué significa el azar para ti? identificando el azar con la palabra homófona "asar", palabra popular en la gastronomía colombiana, mientras que el $10,52 \%$ relaciona el concepto a una situación de suerte. En contraste, la entrevista de salida indica un cambio significativo, donde el $78,94 \%$ de los estudiantes se ubicó en el nivel de desempeño cuatro y cinco (ver tabla 2). Algunas de sus afirmaciones fueron: ningún resultado en un juego de azar es seguro, así mismo, enunciaron ejemplos haciendo referencia a rifas, elecciones escolares, cambios climáticos y juegos como lanzar una moneda para obtener cara o sello. Respecto al ítem 5 (ver tabla 1), en la prueba diagnóstica el $68,42 \%$ de los estudiantes no pudo responder, ya que desconocían la noción de aleatoriedad, mientras que el $26,31 \%$ intentó reconocer sus características sin enunciar un significado preciso. En la entrevista de salida el $72,64 \%$ de la muestra se ubica en los niveles de desempeño cuatro y cinco, mientras que el porcentaje restante se ubica en los niveles dos y tres, situación que refleja una dificultad de comunicación en el aula de clase mas no en la interpretación de los conceptos, lo cual se atribuye a la reciente relación de los estudiantes con estos términos.

Desde este escenario, se sugiere usar el lenguaje básico de la probabilidad desde edades tempranas, como eje transversal del currículo de primaria. En relación a lo anterior, (Ruiz López, 2015) expresa la necesidad de promover el conocimiento básico de conceptos estadísticos y probabilísticos en primaria, debido a la importancia del análisis de datos y de la comprensión de la variabilidad. Dado que la estadística se puede plantear como la ciencia de la decisión y permea las diferentes áreas del conocimiento, su enseñanza debería ser repensada en los diferentes contextos del conocimiento como estrategia de alfabetización estadística. De hecho, algunos estudios, por ejemplo (Rico Romero, 2007), insisten en la necesidad de alfabetizar o desarrollar las competencias estadísticas en los individuos de una comunidad, de forma que las personas estén en capacidad de hacer juicios fundados y tomar decisiones en su vida personal.

Tabla 3. Puntuación obtenida en la entrevista semiestructurada de entrada y de salida.

\begin{tabular}{|c|c|c|c|c|c|c|c|c|c|c|}
\hline \multirow{2}{*}{ 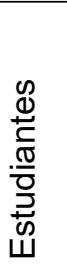 } & \multicolumn{2}{|c|}{ Ítem 1} & \multicolumn{2}{|c|}{ Ítem 2} & \multicolumn{2}{|c|}{ Ítem 3} & \multicolumn{2}{|c|}{ Ítem 4} & \multicolumn{2}{|c|}{ Ítem 5} \\
\hline & 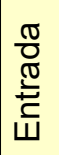 & $\begin{array}{l}\frac{\pi}{0} \\
\frac{.0}{\mathbb{N}} \\
\text { C) }\end{array}$ & 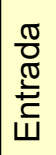 & 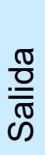 & 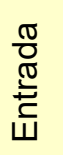 & $\begin{array}{l}\frac{\pi}{\frac{O}{\pi}} \\
\text { ஸे }\end{array}$ & 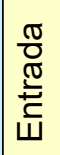 & 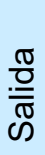 & 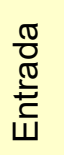 & 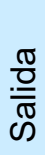 \\
\hline E1 & 2 & 5 & 3 & 4 & 1 & 5 & 2 & 5 & 1 & 4 \\
\hline E2 & 3 & 5 & 3 & 5 & 3 & 4 & 3 & 5 & 2 & 4 \\
\hline E3 & 1 & 4 & 2 & 5 & 1 & 3 & 1 & 5 & 1 & 2 \\
\hline E4 & 1 & 5 & 3 & 5 & 1 & 4 & 1 & 5 & 1 & 4 \\
\hline E5 & 1 & 3 & 1 & 4 & 1 & 4 & 2 & 5 & 1 & 5 \\
\hline E6 & 2 & 5 & 3 & 5 & 1 & 5 & 2 & 5 & 2 & 5 \\
\hline E7 & 1 & 4 & 1 & 5 & 1 & 4 & 1 & 4 & 1 & 3 \\
\hline E8 & 1 & 5 & 3 & 5 & 1 & 5 & 2 & 5 & 1 & 4 \\
\hline
\end{tabular}




\begin{tabular}{|l|l|l|l|l|l|l|l|l|l|l|}
\hline E9 & 2 & 5 & 3 & 5 & 2 & 3 & 1 & 4 & 1 & 3 \\
\hline E10 & 2 & 5 & 2 & 4 & 1 & 5 & 2 & 5 & 1 & 5 \\
\hline E11 & 1 & 4 & 2 & 5 & 1 & 5 & 1 & 5 & 1 & 3 \\
\hline E12 & 1 & 3 & 1 & 4 & 1 & 2 & 1 & 4 & 1 & 3 \\
\hline E13 & 1 & 4 & 2 & 5 & 1 & 4 & 1 & 4 & 1 & 4 \\
\hline E14 & 2 & 5 & 3 & 5 & 2 & 5 & 3 & 5 & 2 & 5 \\
\hline E15 & 2 & 5 & 3 & 5 & 1 & 5 & 3 & 5 & 2 & 4 \\
\hline E16 & 1 & 5 & 2 & 4 & 2 & 3 & 1 & 4 & 1 & 3 \\
\hline E17 & 2 & 4 & 3 & 5 & 2 & 5 & 2 & 5 & 2 & 5 \\
\hline E18 & 2 & 5 & 4 & 5 & 3 & 5 & 4 & 5 & 3 & 5 \\
\hline E19 & 2 & 5 & 3 & 5 & 1 & 5 & 1 & 4 & 1 & 4 \\
\hline
\end{tabular}

Fuente: Elaboración propia

En la anterior tabla se puede observar la comparación entre la puntuación obtenida en las dos entrevistas semiestructuradas implementadas, donde se reconoce el progreso en el desempeño, en el cual, respecto a la entrevista de salida, un número significativo de estudiantes se ubica en los niveles tres, cuatro $y$ cinco.

Se resalta que la motivación por parte de los estudiantes al interactuar con los recursos virtuales disponibles en la página web fue mayor al grado de motivación experimentado en las clases clásicas presenciales. Por consiguiente, la situación de confinamiento brinda una gran oportunidad de innovación en las prácticas pedagógicas del contexto rural.

\section{CONCLUSIONES $Y$ RECOMENDACIONES}

Dado el impacto favorable de implementar los recursos TIC en la escuela rural, desde el campo de la didáctica de la estadística, es importante seguir articulando el desarrollo de las competencias estadísticas con el uso de recursos tecnológicos en la post-pandemia, debido a su influencia en la motivación de los estudiantes y la pertinencia de su uso en la actualidad. Sin embargo, el docente debe tener en cuenta que tanto los aparatos tecnológicos como la conectividad a internet en la ruralidad es limitada $y$, por tanto, se recomienda el uso simultáneo de material impreso para las actividades escolares de tal manera que sirva de apoyo en el momento que no sea posible acceder a los recursos virtuales.

En este escenario, es importante la autocapacitación docente y el mejoramiento de sus habilidades en el uso de recursos tecnológicos, lo que puede incidir significativamente en el diseño e implementación de secuencias didácticas articuladas a las TIC. Es claro que la buena actitud que asume el docente frente a los retos educativos que se derivan de las situaciones de emergencia se contagia a los estudiantes, esto permite ver las oportunidades que se presentan y vencer las limitaciones y dificultades propias de los tiempos difíciles.

Por otra parte, el desarrollo de cada una de las fases investigativas permite evidenciar un aprendizaje significativo de la probabilidad intuitiva en estudiantes de grado tercero primaria de la institución rural mencionada, aunado a ello, se afirma que el uso de situaciones cotidianas, la construcción del material didáctico por parte del estudiante, las actividades de gamificación y el uso de un lenguaje menos formal que no cambie el significado de los términos estadísticos son ingredientes de una receta que potencia la capacidad de comprensión de la probabilidad intuitiva y que favorece el razonamiento crítico de los estudiantes.

Debido a que los padres de familia juegan un rol más activo en el proceso escolar de sus hijos, algunas actividades pueden ser repensadas con el lente del fortalecimiento de las relaciones emocionales, el compromiso mutuo en el proceso educativo y la alfabetización estadística.

En las zonas rurales se aconsejan los encuentros sincrónicos cortos vía WhatsApp de manera individual $\mathrm{o}$ en pequeños grupos, siempre y cuando se pueda garantizar la conectividad a internet y la disponibilidad del hardware. En este caso, por lo general, los educandos utilizaron 
recargas de datos en la Sim Card del teléfono inteligente disponible en casa.

\section{REFERENCIAS BIBLIOGRÁFICAS}

Ander-Egg. E. (2003). Repensando la Investigación-Acción Participativa. Lumen Hvmanitas. Obtenido de https:// s3.amazonaws.com/academia.edu. documents

Burbano Pantoja, V., Valdivieso Miranda, M., \& Aldana Bermúdez, E. (2017). Conocimiento base para la enseñanza: un marco aplicable en la didáctica de la probabilidad. Revista de investigación, desarrollo e innovación, Vol.7, $\mathrm{N}^{\circ}$. 2, págs. 269-285. doi:10.19053/20278306. v7.n2.2017.6070

Consejería de Educación de la Junta de Castilla y León. (2015). Las nuevas metodologías en la enseñanza y el aprendizaje de las Matemáticas. Segovia: Academia de Artillería de Segovia. Obtenido de https://www.researchgate.net/ publication/316989920

Cuevas Solano, E. (2020). Una puerta abierta a la aventura de la probabilidad. Obtenido de webnode: https://explorando-elmundo-de-la-probabilidad2.webnode. com.co

Gómez Torres, E., Contreras, J., \& Batanero, C. (Septiembre de 2015). Significados de la probabilidad en libros de texto para educación primaria en Andalucía. (C. Fernández, M. Molina, \& N. Planas, Edits.) Obtenido de http://hdl.handle. net/10045/51378

Hernández Sampieri, R., Fernández Collado, C., \& Baptista Lucio, P. (2004). Metodología de la Investigación. México: McGrawHill Interamericana.
ICFES. (07 de Septiembre de 2015). SABER $3^{\circ}, 5^{\circ}, 9^{\circ}$ (APLICACIONES ANTES DE 2018). Obtenido de https://www.icfes. gov.co/web/guest/resultados-historicossaber-359

Jiménez Vargas, J. (2014). Diseño y Planificación de la noción de Azar y Probabilidad en Educación Primaria. TFG de Maestro de Educación Primaria. España: Universidad de Cadiz. Obtenido de https://rodin.uca.es/xmlui/ handle/10498/16628

Ministerio de Educación Nacional. (2016). Colombia Aprende. Obtenido de Derechos Básicos de Aprendizaje: http://aprende.colombiaaprende.edu. co/sites/default/files/naspublic/DBA_ Matem\%C3\%A1ticas.pdf

Molina Bernal, I. A., Morales Piñero, J. C., Rodríguez Jerez, S. A., Cote Sánchez, M. C., Martínez Molina, B., Martínez León, H., . . . Rojas T, E. L. (2019). Importancia de las TIC en los procesos de enseñanza-aprendizaje: estudios de la educación media y superior. doi:10.22518/book/9789585511743

Pandemia de COVID-19. (27 de abril de 2021). Wikipedia, La enciclopedia libre. Fecha de consulta: 23:57, abril 27, 2021 Obtenido de https://es.wikipedia.org/w/ index.php?title=Pandemia_de_COVID19\&oldid=135092509

Rico Romero, L. (2007). La Competencia Matemática en PISA. Revista PNA, Vol. 1, No. 2. Obtenido de https://dialnet.unirioja.es/servlet/ articulo?codigo $=2238336$

Ruiz López, N. (2015). La enseñanza de la Estadística en Educación Primaria en América Latina. Revista Iberoamericana sobre Calidad, Eficacia y Cambio en 
Educación, Vol. 13, №. 1, págs. 103121. Obtenido de http://www.rinace.net/ reice/numeros/arts/vol13num1/art6.pdf

Tobón Tobón, S., Pimienta Prieto, J., \& García Fraile, J. (2010). Secuencias didácticas: aprendizaje y evaluación de competencias, Vol. 10, №. 31. Obtenido de https://www.pearsoneducacion.net/ 